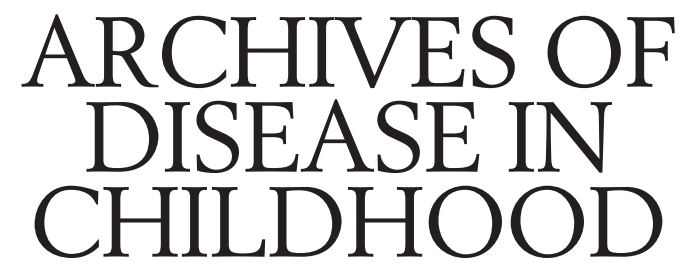

The Fournal of the Royal College of Paediatrics and Child Health

\title{
EDITORIAL
}

\section{Aging and paediatric practice}

\author{
Malcolm L Chiswick, Harvey Marcovitch
}

Scientific journals from around the world have agreed to take part this year in a global themed issue on aging - an initiative coordinated by $\mathcal{F} A M A$. How do we justify a contribution from the Archives of Disease in Childhood to this important theme? One thing that distinguishes paediatric practice is that health and disease are observed against a background of growth and development. Perhaps analogous to this is the practice of geriatrics, where biological senescence, and all its psychosocial implications, is the backdrop against which health and disease are observed. Yet this philosophy becomes clouded when we consider that development and aging (or senescence) cannot be readily pulled apart. Although development implies an advantageous adaption to life, and aging implies irreversible deterioration it is none the less true that development is not only a precursor of aging but also influences it profoundly.

If we are serious about health promotion as a science we need to have a better understanding of the factors that govern the various markers of morbidity in adult life, such as obesity. The relationship between parental obesity and obesity in childhood is well known. In this issue, fulie Lake and colleagues (see p 376), using longitudinal data from the 1958 British birth cohort, show that tracking of body mass index (BMI) from childhood to adult life occurred and was strongest among those whose both parents were obese; indeed, such children when they reached the age of 33 years had a mean BMI 20\% greater than those in whom both parents were 'normal'. They warn that a rising prevalence of adult obesity is likely to strengthen the tracking of child to adult BMI in the next generation.

Obesity of central distribution in adults is a feature of non-insulin dependent diabetes and insulin resistance. There is a link between this syndrome and low birth weight. This has prompted Mary Barker and colleagues to exam- ine the association between birth weight and body fat distribution in adolescent girls. Their observations, described on $\mathrm{p} 381$, lend support to the suggestion that a tendency to store fat on the trunk in overweight adolescent girls may be programmed by growth, or more precisely by the lack of it, in fetal life.

It is likely that improved methods of epidemiological research will reveal numerous curious and provocative associations between health experiences in early childhood and the occurrence of later disease. Reduced exposure to infection in childhood has been implicated as a factor in the pathogenesis of insulin dependent diabetes (IDDM). Caspar Gibbon et al put this theory to the test in a brief but important paper on $\mathrm{p} 384$ which points to a relationship between decreased exposure to common infections in infancy, based on general practitioner records, and IDDM in children under the age of 16 years.

In this months Fetal and Neonatal Edition we continue the theme with a review article by $A$ Aihie Sayer et al who pose the somewhat depressing question: 'Is lifespan determined in utero?' (see p F162). It is not surprising that it might be, given the association between poor fetal growth and an increased risk of cardiovascular disease in adults. However, these authors draw attention to poor growth in fetal life and infancy, and the promotion of biological senescence in systems such as the eye, ear, muscle, and skin. They speculate that nutritional programming of aging might come about through impaired development of tissue repair systems at cellular and molecular levels.

Programmed cell death (apoptosis), unlike necrosis, is characterised by preservation of membrane integrity and organelle structure, the occurrence of chromatin condensation and nuclear fragmentation, and the budding off of cellular fragments known as apoptotic bodies, from the Greek apo (away from) and ptosis
Childhood, BMA

House, Londo WC1H 9JR 
(falling). This process occurs in diverse conditions, some physiological, such as in embryonic development and involutional processes; and others pathological, as in immune mediated cell killing, and ischaemic injury. In their review article Nicholas D Mazarakis and colleagues (see p F165) highlight the broad spectrum of neurological disorders where apoptosis is a feature, including hypoxicischaemic brain injury in the newborn. The importance of learning more about this form of cell death is that if effective antiapoptotic strategies were available they would be potentially applicable to a wide range of disorders.

If, as we have seen, fetal nutrition influences health in adulthood it is reasonable to ask to what extent aging, in this context biological senescence, occurs in the placenta. This subject is reviewed by Harold Fox (see pF171) who indicates that, far from senescence, the term placenta shows continuing DNA synthesis, and persisting villous growth and expansion of villous surface area. He refutes the widely held notion that, come 40 weeks' gestation, the placenta goes into a decline, both functionally and anatomically. Indeed, he draws attention to fetal macrosomia in prolonged pregnancy. $\mathrm{He}$ argues that the classical clinical syndrome of 'postmaturity' (now uncommon) is related to oligohydramnios, for which the placenta should not take the blame as there is no evidence that in late pregnancy it has a role in the production of amniotic fluid, or in the control of its volume.

In conclusion, high quality epidemiological research during the next decade will present us with many intriguing discoveries about the influence of fetal and early childhood experience on diseases in adult life. We need also to tease out similarities of biological mechanisms of disease processes in the young and the elderly. Treatment strategies aimed at manipulating biological processes may be common to both. Stroke in the elderly and hypoxicischaemic brain damage in the newborn is but one example. We should also acknowledge the notion that 'dependency' provokes similar psychosocial problems at the two extremes of life including neglect and abuse. The basic science that governs the state of dependency of the very young and the elderly has similarities that might be exploited to provide improved preventive and management strategies for both, even though there will be differences in detail.

\title{
Child to adult body mass index in the 1958 British birth cohort: associations with parental obesity
}

\author{
Julie K Lake, Chris Power, Tim J Cole
}

\section{Department of Epidemiology and Public Health, Institute of Child Health, 30 Guilford Street, London WC1N 1EH \\ J K Lake \\ C Power}

MRC Dunn Nutrition Unit, Cambridge T J Cole

Correspondence to: J K Lake.

\begin{abstract}
Objectives-To assess relations between the adiposity of children and their parents and to establish whether tracking of adiposity from childhood to adulthood varies according to the parental body mass index (BMI).

Methods-Longitudinal data from the 1958

British birth cohort study were used (6540 men and 6207 women). The height and weight of the study subjects were measured at $7,11,16,23$ (self reported), and 33 years. Parental height and weight were self reported when their children were 11 years old. The children were classified into six parental BMI (weight/height ${ }^{2}$ ) groups.

Results-At each age of follow up the mean BMI of the children increased as the parental BMI increased. Higher risks of adult (33 year) obesity were evident among children with overweight or obese parents: the odds for sons and daughters with two obese parents (compared with those with both parents of normal BMI)
\end{abstract}

were 8.4 and 6.8 , respectively. The children of two obese parents also showed the strongest child to adult tracking of BMI as indicated by the correlation between ages 7 and $33(r=0.46,0.54$, sons and daughters, respectively).

Conclusions-The children of obese and overweight parents have an increased risk of obesity. Subjects with two obese parents are fatter in childhood and also show a stronger pattern of tracking from childhood to adulthood. As the prevalence of parental obesity increases in the general population the extent of child to adult tracking of BMI is likely to strengthen. (Arch Dis Child 1997;77:376-381)

Keywords: body mass index; child to adult tracking; obesity; parent

Obesity is a common nutritional disorder and is an important risk factor for several chronic diseases. ${ }^{12}$ Thus factors influencing the 